\title{
Estoque de árvores em uma área não manejada na Floresta Nacional do Tapajós, PA
}

\author{
Daniele Lima da Costa ${ }^{1}$ Misael Freitas dos Santos ${ }^{1}$ Lia Oliveira Melo ${ }^{2}$ João Ricardo Vasconcellos Gama ${ }^{2}$ \\ Talita Godinho Bezerra ${ }^{3}$ Bruno de Almeida Lima ${ }^{4}$ Girlene da Silva Cruz ${ }^{4}$ Bruno Rafael Silva de Almeida ${ }^{4}$ \\ ${ }^{1}$ Programa de Pós-Graduação em Ciências Florestais (PPGCF), Universidade Estadual do Centro Oeste, Rodovia PR 153, km 7, Riozinho, Irati-PR, 84500-000 \\ 2 Programa de Pós-Graduação em Sociedade, Natureza e Desenvolvimento, Universidade Federal do Oeste do Pará, Rua Vera Paz, s/n, Salé, Santarém-PA, \\ 68040-255 \\ ${ }^{3}$ Programa de Pós-Graduação em Ciências Florestais, Instituto de Ciências Agrárias, Universidade Federal Rural da Amazônia, Av Presidente Tancredo Neves, \\ 2501, Terra Firme, Belém-PA, 66.077-830 \\ ${ }^{4}$ Programa de Pós-Graduação em Engenharia Florestal (PPGEF), Universidade do Estado de Santa Catarina, Centro de Ciências Agroveterinárias, Av. Luiz de \\ Camões, 2090 - Conta Dinheiro, Lages - SC, 88520-000
}

*Author for correspondence: danielelimadacosta@gmail.com

Received: November 2018 / Accepted: February 2019 / Published: June 2019

\section{Resumo}

O presente estudo objetivou analisar a estrutura populacional e o estoque dos recursos florestais de uma área não manejada na Floresta Nacional do Tapajós, estado do Pará. Para isso, foram alocadas sistematicamente 67 unidades amostrais com $20 \mathrm{~m}$ x 100 $\mathrm{m}$, onde foram mensuradas todas as árvores com diâmetro à altura do peito (DAP) $\geq 5 \mathrm{~cm}$, considerando as seguintes classes de tamanhos (CT): CT 1-Regeneração natural $(20 \mathrm{~m} \mathrm{x} 10 \mathrm{~m}): 5 \mathrm{~cm} \leq \mathrm{DAP}<10 \mathrm{~cm}$; CT $2-$ Futura colheita $(20 \mathrm{~m} \times 50 \mathrm{~m}): 10 \mathrm{~cm} \leq$ DAP $<50 \mathrm{~cm}$; CT 3-Colheita $(20 \mathrm{~m} \times 100 \mathrm{~m}):$ DAP $\geq 50 \mathrm{~cm}$. Foram avaliados a estrutura populacional, distribuição diamétrica e espacial, qualidade de fuste das árvores e a presença de cipó titica (Heteropsis spp). As espécies Carapa guianensis, Lecythis lurida e Manilkara elata que possuem alto interesse comercial estiveram entre as de maiores importância na área e com maiores volumes. Os tipos de distribuição espacial abrangeram totais de árvores bastante próximos e a distribuição diamétrica para a floresta e para as três espécies de interesse comercial seguiu o formato de " $\mathrm{j}$ " invertido. A área em estudo sugere potencial de recursos madeireiros e não madeireiros, com boa ocorrência de cipó titica como fonte potencial de utilização por comunidades, elucidando que o uso múltiplo de florestas deve ser considerado e estudos que mostrem esta viabilidade devem ser incentivados e replicados na Amazônia.

Palavras-chave: Fitossociologia, Distribuição diamétrica, Distribuição Espacial, Amazônia.

\begin{abstract}
The objective of this study was to analyze the population structure and the stock of forest resources in an unmanaged area in the Tapajós National Forest, in the state of Pará. For this, 67 sample units with 20 mx $100 \mathrm{~m}$ were systematically allocated, where they were measured all trees with diameter at breast height $(\mathrm{DBH}) \geq 5 \mathrm{~cm}$, considering the following size classes (CT): CT 1-Natural regeneration $(20 \mathrm{mx} 10 \mathrm{~m}): 5 \mathrm{~cm} \leq \mathrm{DBH}<10 \mathrm{~cm}$; CT 2Future harvest $(20 \mathrm{~m} \mathrm{x} 50 \mathrm{~m}): 10 \mathrm{~cm} \leq \mathrm{DBH}<50 \mathrm{~cm}$; CT 3 -Harvest $(20 \mathrm{~m} \times 100 \mathrm{~m}): \mathrm{DBH} \geq 50 \mathrm{~cm}$. The floristic composition, population structure, diameter and spatial distribution, tree stem quality and the presence of vine (Heteropsis spp.) were evaluated. The species Carapa guianensis, Lecythis lúrida and Manilkara elata that have high commercial interest were among the most important in the area and with higher volumes. The types of spatial distribution encompassed totals of trees fairly close
\end{abstract}

together and the diametric distribution to the forest and to three species of commercial interest followed the inverted "J" shape. The study area has potential for timber and non-timber resources, with a good occurrence of Heteropsis spp., elucidating that the multiple use of forests should be considered and studies that show this viability should be encouraged and replicated in the Amazon.

Keys-worlds: Phytosociology, Diametric Distribution, Spatial Distribution, Amazonia.

\section{Introdução}

As florestas nativas do bioma amazônico são conhecidas mundialmente pela sua exuberante biodiversidade, bem como por sua grande extensão territorial e pelas diversas ações antrópicas que tem sofrido (Andrade e Manzatto 2014). Contudo, a maioria das florestas tropicais nativas da Amazônia tem sido explorada de forma convencional, sem aplicação dos critérios de sustentabilidade do manejo florestal, o que pode ocasionar o desequilibro ecológico da floresta e, consequentemente, a perda da cobertura florestal e da diversidade de espécies, podendo comprometer a perpetuidade deste ecossistema (Lima-Filho et al. 2002; Souza et al. 2006; Callegaro et al. 2012).

Neste cenário, a avaliação dos fatores estruturantes da floresta como composição florística, estrutura fitossociológica e diamétrica, tornam-se imprescindíveis para o planejamento e o estabelecimento de processos chave para a manutenção da floresta e a conservação da biodiversidade como, por exemplo, a determinação do potencial florestal e o aproveitamento dos recursos a partir de sistemas de manejo com produção sustentável (Oliveira e Amaral 2004; Gama et al. 2005; Silva-Júnior 2005; Souza et al. 2006; Lima et al. 2012).

Outra informação de grande importância para a avaliação e caracterização dos recursos existentes em determinada área de floresta nativa é a forma como as árvores estão distribuídas espacialmente. $\mathrm{O}$ padrão de distribuição espacial pode ser caracterizado como o arranjo dos membros de uma população em um habitat, que pode ocorrer de forma aleatória, uniforme ou agregada (Begon et al. 2006). Esse comportamento é resultante da ação conjunta de fatores abióticos e bióticos, como competição por espaço, disponibilidade de nutrientes, luz e água sobre os processos de recrutamento e mortalidade (Lehn e Resende 2007).

Contudo, é extremamente necessário obter estimativas confiáveis das características da população de 
interesse (Souza et al. 2006; Soares et al. 2009). Os resultados desses processos devem permitir estimativas confiáveis de variáveis dendrométricas, bem como representar suficientemente a composição de espécies das comunidades vegetais amostradas. Neste contexto, o presente estudo teve como objetivo analisar a estrutura populacional e o estoque de recursos florestais de uma área não manejada na Floresta Nacional do Tapajós, PA.

\section{Material e métodos \\ Área de Estudo}

O inventário florestal foi realizado em uma área de 100 ha localizada na Floresta Nacional do Tapajós (Flona do Tapajós), Km 72 da Rodovia BR-163 (SantarémCuiabá), no município de Belterra, no estado do Pará (Figura 1). O clima da região é do tipo Am, segundo Alvares et al. (2013). A vegetação característica da região é do tipo Floresta Ombrófila Densa de terra firme (IBGE 2012).

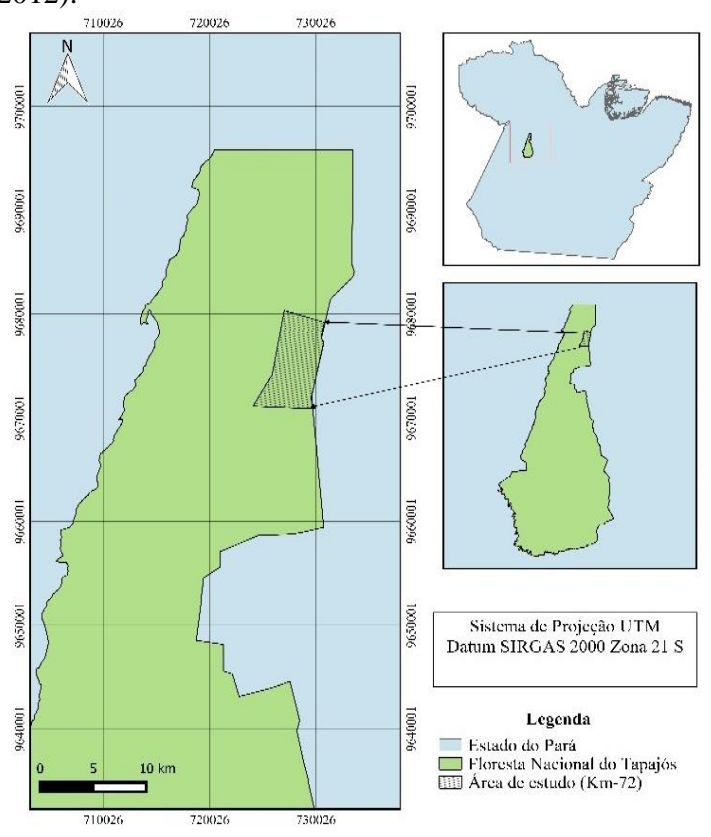

Figura 1. Localização da área de estudo na Floresta Nacional do Tapajós, PA.

\section{Amostragem e coleta de dados}

Foram estabelecidas 67 parcelas de $20 \mathrm{~m}$ x $100 \mathrm{~m}$ de maneira sistemática na área, totalizando um esforço amostral de 13,4 ha. As parcelas foram subdividas em três níveis de inclusão: Regeneração natural - CT 1 (20 $\mathrm{m} \times 10 \mathrm{~m}$ ): $5,0 \mathrm{~cm} \leq \mathrm{DAP}<10,0 \mathrm{~cm}$; Futura colheita CT $2(20 \mathrm{~m} \times 50 \mathrm{~m}): 10,0 \mathrm{~cm} \leq \mathrm{DAP}<50,0 \mathrm{~cm}$; Colheita - CT $3(20 \mathrm{~m} \times 100 \mathrm{~m}):$ DAP $\geq 50,0 \mathrm{~cm}$.

Nas parcelas foram obtidas a circunferência à altura do peito (CAP) maior ou igual a $15,8 \mathrm{~cm}$, altura comercial (hc), qualidade de fuste (QF) e presença de cipó Heteropsis spp. comercialmente chamado de cipótitica. A qualidade de fuste foi classificada da seguinte forma: Qualidade de fuste $1(\mathrm{QF} 1)$, árvores que não apresentavam tortuosidade ao longo de seu fuste; Qualidade de fuste $2(\mathrm{QF} 2)$, árvores que apresentavam pouca tortuosidade ao longo do fuste; e, Qualidade de fuste 3 (QF3), árvores que apresentavam bastante tortuosidade ao longo de seu fuste.

As espécies foram identificadas na floresta com seus nomes vernaculares por parataxonomistas. As árvores que suscitaram dúvidas tiveram sua identificação taxonômica feita por meio de comparações de exsicatas no herbário da Universidade Federal do Oeste do Pará UFOPA. As atualizações da nomenclatura botânica foram realizadas por meio de consulta ao banco de dados do Portal Reflora (FLORA DO BRASIL, 2018).

\section{Análise dos dados Estrutura horizontal}

A estrutura horizontal foi descrita por meio dos parâmetros de densidade, dominância e frequência de acordo com as fórmulas descritas por Souza e Soares (2013). Para determinar o índice de valor de importância (VI) foi adotada a metodologia proposta por Salomão (2012).

\section{Distribuição diamétrica e volumétrica}

A distribuição diamétrica da floresta foi projetada para todas as árvores inventariadas e para as espécies com maiores valores de importância que apresentam interesse comercial. Determinou-se a distribuição das árvores em classes diamétricas pela relação entre o número de árvores por hectare e centros de classes diamétricas. Considerando todas as árvores inventariadas a distribuição foi realizada para as árvores com $5 \mathrm{~cm} \leq$ DAP $>25 \mathrm{~cm}$, com amplitude de 2,5 $\mathrm{cm}$ e para as árvores com DAP $\geq 25 \mathrm{~cm}$, com amplitudes de $10 \mathrm{~cm}$. A distribuição volumétrica foi realizada considerando o volume das árvores agrupadas nos centros de classes diamétricas, sendo o volume estimado pela seguinte fórmula (Equação 1):

$$
\mathrm{Vf}=\frac{\pi^{*} \mathrm{DAP}^{2}}{400000} * \mathrm{~h} * \mathrm{ff} \quad \text { Equação (1) }
$$

Sendo: $\mathrm{Vf}=$ volume de fuste com casca, $\mathrm{em}^{3}$; $\mathrm{DAP}=$ diâmetro medido a $1,30 \mathrm{~m}$ do solo, em $\mathrm{cm}$; $\mathrm{h}=$ altura total, em $\mathrm{m}$; e ff = fator de forma, sendo utilizado 0,7 (Heinsdijk e Bastos 1963; SEMAS 2015).

\section{Distribuição espacial}

Para a análise da distribuição espacial das árvores, utilizou-se o Índice de Morisita, conforme recomendado por Brower e Zar (1977), por ser pouco influenciado pelo tamanho da unidade de amostra e apresentar excelente qualidade na detecção do grau de dispersão, determinado pela equação 2 (Calegário et al. 1993; Bentes-Gama et al. 2002; Vieira et al. 2014):

$$
\mathrm{IM}=\frac{\mathrm{n}^{*}\left(\sum \mathrm{X}^{2}-\mathrm{N}\right)}{\mathrm{n}(\mathrm{N}-1)} \quad \text { Equação (2) }
$$

Sendo: $\mathrm{IM}=$ Índice de Morisita da i-ésima espécie; $\mathrm{n}=$ número total de parcelas amostradas; $\mathrm{N}=$ número total de árvores, contidas nas $\mathrm{n}$ parcelas; $\mathrm{X}^{2}=$ quadrado do número de árvores por parcela.

Se IM $=1,0$, infere-se que a i-ésima espécie tem padrão de distribuição espacial aleatório; se IMi < 1,0, o padrão de distribuição espacial da i-ésima espécie é rara; e se IMi > 1,0, o padrão de distribuição é agregado. O nível de significância dos valores de IM foi obtido pelo teste Qui-quadrado (Equação 3)

$$
\mathrm{X}^{2}=\frac{\mathrm{n}^{*} \sum \mathrm{X}^{2}}{\mathrm{~N}}-\mathrm{N} \quad \text { Equação (3) }
$$

Sendo: $X^{2}=$ valor de qui-quadrado; e $n, N, s, X^{2}$ já definidos anteriormente.

\section{Qualidade de fuste e ocorrência de cipó titica (Heteropsis spp.)}

As árvores foram contabilizadas de acordo com a classificação de qualidade de fuste. Para análise da ocorrência de Heteropsis spp., foi obtido o total de 
árvores hospedeiras e as principais espécies em que se encontrou o hospedeiro. Estimou-se também a distribuição deste cipó por classes de alturas das árvores hospedeiras.

\section{Resultados e discussão Estrutura horizontal}

A densidade de indivíduos estimada foi de 659,70 arv.ha ${ }^{-1}$, representando uma área basal de $20,77 \mathrm{~m}^{2} \cdot \mathrm{ha}^{-1}$. Desse total, o nível de regeneração natural $(5,0 \leq \mathrm{DAP}<$ $10,0)$ registrou 341,79 arv.ha $^{-1}$; o de futura colheita $(10,0$ $\leq$ DAP $<50,0) 296,42$ arv.ha ${ }^{-1}$; e o nível de colheita (DAP $\geq 50,0) 21,49$ arv.ha $^{-1}$. Para as árvores com o limite de inclusão de DAP $\geq 10 \mathrm{~cm}$, a densidade estimada foi de 317,51 arv $\cdot$ ha $^{-1}$. Gonçalves e Santos (2008) ao estudarem a composição florística e estrutural de uma área de manejo também na Flona do Tapajós encontraram uma densidade de 369 arv.ha $^{-1}$ e área basal de 22,5 m².ha ${ }^{-1}$. Entretanto, os autores consideraram todos os indivíduos arbóreos com DAP $\geq 10 \mathrm{~cm}$ em uma área amostral de 6 ha, menor que a relatada no presente estudo.

As dez espécies que ocorreram com maior frequência na área foram Carapa guianensis Aubl., Couratari sp., Virola duckei A.C.Sm., Manilkara elata (Allemão ex Miq.) Monach., Tachigali myrmecophila (Ducke) Ducke, Coussarea paniculata (Vahl) Standl., Maquira sclerophylla (Ducke) C.C.Berg, Ocotea neesiana (Miq.) Kosterm., Tachigali chrysophylla (Poepp.) Zarucchi \& Herend. e Lecythis lurida (Miers) S.A.Mori. Foi observado que $90 \%$ das espécies mais frequentes também apresentaram os maiores valores de importância (Tabela 1). Dentre estas, destacaram-se espécies de grande aceitação no mercado madeireiro da região como Couratari sp., Manilkara elata e Lecythis lurida, apreciadas pelo seu valor comercial e qualidade da madeira.

A espécie Carapa guianensis se destacou como a espécie de maior VI. Esta é uma espécie de uso múltiplo para o mercado de produtos não madeireiros, devido os recursos que proporciona, tais como a madeira, frutos com sementes, de onde é obtido um óleo utilizado para diversas finalidades medicinais e também sua casca que é utilizada na medicina popular (Shanley 2005). Esta espécie tem grande importância como fonte de renda em comunidades tradicionais (Mendonça e Ferraz 2007).

Tabela 1. Estrutura horizontal das 10 espécies com maiores valores de importância (VI). Em que: DA: Densidade Absoluta; DR: Densidade Relativa; FA: Frequência Absoluta; FR: Frequência Relativa; DoA: Dominância Absoluta; DoR: Dominância Relativa; VC: Valor de Cobertura; VI: Valor de Importância (\%).

\begin{tabular}{|c|c|c|c|c|c|c|c|c|}
\hline $\begin{array}{ll}\text { Espécie } \\
\end{array}$ & DA & DR & FA & FR & DoA & DoR & $\overline{\mathrm{VC}}$ & $\bar{\nabla}$ \\
\hline Carapa guianensis Aubl. & 12,31 & 1,87 & 68,66 & 2,61 & 1,44 & 6,91 & 4,39 & 3,80 \\
\hline Virola duckei A.C.Sm & 21,57 & 3,27 & 65,67 & 2,49 & 0,83 & 3,99 & 3,63 & 3,25 \\
\hline Couratarisp. & 18,81 & 2,85 & 65,67 & 2,49 & 0,75 & 3,60 & 3,23 & 2,98 \\
\hline Coussarea paniculata Standl. & 31,79 & 4,82 & 56,72 & 2,15 & 0,25 & 1,19 & 3,01 & 2,72 \\
\hline Tachigali myrmecophila (Ducke) Ducke & 13,88 & 2,10 & 58,21 & 2,21 & 0,78 & 3,74 & 2,92 & 2,69 \\
\hline Manilkara elata (Allemão ex Miq.) Monach. & 9,40 & 1,43 & 58,21 & 2,21 & 0,80 & 3,83 & 2,63 & 2,49 \\
\hline Lecythis lurida (Miers) S.A.Mori & 6,72 & 1,02 & 47,76 & 1,81 & 0,94 & 4,54 & 2,78 & 2,46 \\
\hline $\begin{array}{l}\text { Tachigali chrysophylla (Poepp.) Zarucchi \& } \\
\text { Herend. }\end{array}$ & 11,34 & 1,72 & 52,24 & 1,98 & 0,74 & 3,57 & 2,64 & 2,42 \\
\hline Protium paniculatum Engl. & 20,45 & 3,10 & 46,27 & 1,76 & 0,34 & 1,63 & 2,37 & 2,16 \\
\hline Maquira sclerophylla (Ducke) C.C.Berg & 15,45 & 2,34 & 55,22 & 2,10 & 0,40 & 1,94 & 2,14 & 2,13 \\
\hline Subtotal & 180,15 & 27,31 & 619,40 & 23,53 & 7,63 & 36,71 & 32,01 & 29,11 \\
\hline Total & 659,70 & 100,00 & 2632,84 & 100,00 & 20,77 & 100,00 & 100,00 & 100,0 \\
\hline
\end{tabular}

\section{Distribuição espacial}

A análise da distribuição espacial permitiu verificar que as espécies raras representam $33,78 \%$ do total. As demais, 30,41\% se distribuem de forma aleatória; 18,58
$\%$ de forma agregada e $17,23 \%$ tendem ao agrupamento. As espécies com os maiores VI apresentaram-se distribuídas em tendência a agregação e com ocorrência de forma agrupada.

As espécies Carapa guianensis e Lecythis lúrida se distribuem espacialmente na área com tendência a formar agrupamentos e as espécies Couratari sp. e Manilkara elata ocorrem com distribuição agrupada. $\mathrm{O}$ fato das espécies com grande interesse comercial se distribuírem com tendência ao agrupamento e de forma agrupada, poderá facilitar possíveis operações de colheita de madeira na área, bem como, a coleta de frutos para produção de óleo, como o caso da espécie Carapa guianensis.

A distribuição espacial apresentada pelas espécies reflete a influência das diferentes variáveis ambientais existentes em cada sítio (Camarero et al. 2000), o tipo de dispersão de sementes que cada espécie tem e a competição intraespecífica e interespecífica (Capretz 2004). O total de espécies raras foi bastante próximo ao de espécies distribuídas aleatoriamente, sendo que espécies com distribuição agregada e aquelas com tendência a agregação, juntas, também somam um total de árvores próximo das demais distribuições.

A informação sobre a raridade de uma espécie, implica diretamente em sua vulnerabilidade, sendo que os casos de raridade para as espécies florestais podem ser extremos ou mais brandos. Em geral, são influenciados pelas características individuais de cada espécie, como sua biologia reprodutiva, capacidade de dispersão, habilidade de competição, que podem interagir como o meio abiótico e ocasionar diferentes cenários de espacialização das espécies (Fontana e Sevegnani, 2012).

\section{Distribuição diamétrica}

A distribuição do número de árvores por classe diamétrica, com amplitude de $10 \mathrm{~cm}$, seguiu o padrão característico das florestas inequiâneas, ou seja, distribuição exponencial negativa, na forma de "J" invertido (Figura 2). Nota-se que as árvores com $5 \mathrm{~cm} \leq$ DAP > $25 \mathrm{~cm}$ são as de maiores ocorrência. De maneira geral, as espécies apresentam boa regeneração $(5 \mathrm{~cm} \leq$ DAP $<10 \mathrm{~cm})$ e estoque de crescimento $(10 \mathrm{~cm} \leq \mathrm{DAP}<$ $50 \mathrm{~cm}$ ), o que poderia favorecer o manejo florestal na área.

Este mesmo padrão de distribuição também foi encontrado por Vieira et al. (2014) na comunidade de Santo Antônio (PA); Ribeiro et al. (2013) no município de Placas (PA); Souza et al. (2006) em Paragominas (PA); Andrade et al. (2015) e Gonçalves e Santos (2008) na Flona do Tapajós. Estes últimos ressaltam que isto pode ocorrer devido à existência de um balanço entre o recrutamento e a mortalidade de árvores na área.

A distribuição diamétrica das espécies de bom reconhecimento no mercado florestal da região, dentre as espécies de maiores valores de importância, estão na figura 4. As mesmas estão inseridas no mercado regional pela sua importância madeireira e não madeireira, como o caso de Carapa guianensis. Estas espécies tem uma tendência a distribuição no formato " $\mathrm{j}$ " invertido. Diferentemente da distribuição encontrada para todas as espécies da área, a análise em separado para as três espécies mostrou que a densidade ao longo dos centros de classes não apresenta um decréscimo contínuo. Para estas espécies, algumas classes decrescem mais acentuadamente e, no caso da espécie Lecythis lúrida, o centro de classe $85 \mathrm{~cm}$ não apresentou árvores. 

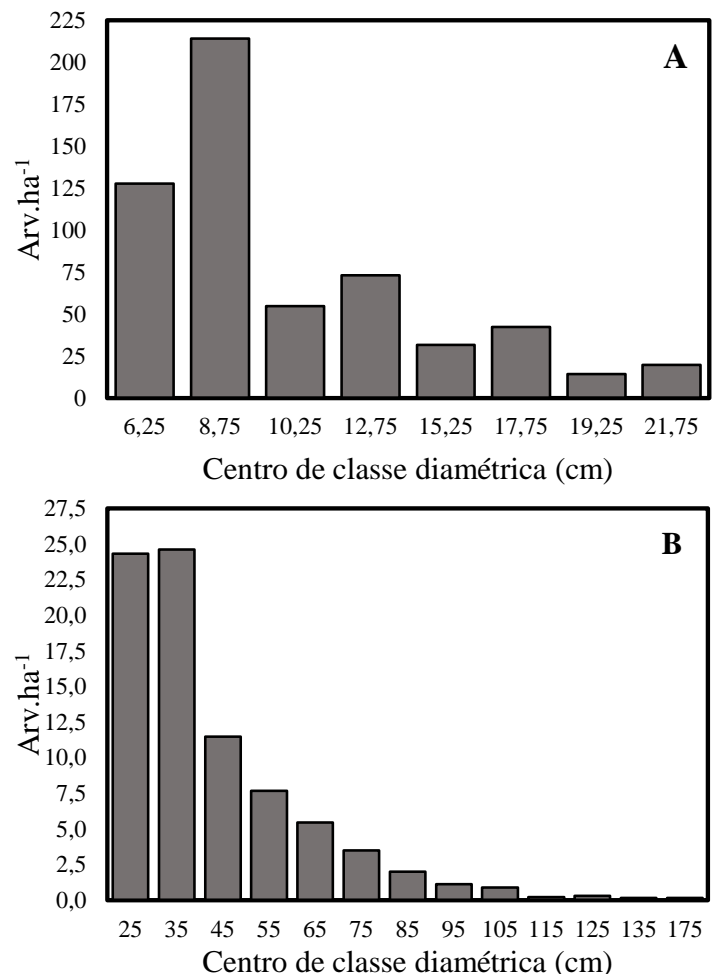

Figura 2. Distribuição diamétrica dos indivíduos com $5 \mathrm{~cm} \leq$ DAP $<25 \mathrm{~cm}$ (A) e DAP $\geq 25 \mathrm{~cm}$ (B) na Floresta Nacional do Tapajós, PA.

A distribuição diamétrica de Lecythis lúrida (Figura 3 A) apresenta irregularidade com a falta de árvores no centro de classe $85 \mathrm{~cm}$. Este fato demonstra que sua população está em desiquilíbrio entre as classes diamétricas. De acordo com Reinhardt et al. (2007) alguns fatores podem explicar certas irregularidades na distribuição diamétrica de espécies, sendo os aspectos relacionados a fisiologia das árvores tropicais bastante preponderante.

A espécie Manilkara elata apresentou maior decréscimo na segunda classe diamétrica em relação à primeira e baixíssimas densidades nas classes seguintes (Figura $3 \mathrm{~B}$ ), podendo sugerir que suas plântulas e árvores ingressantes no estrato arbóreo tiveram dificuldades para se desenvolver e permanecer na floresta. Segundo Costa et al. (2007), esta espécie é tolerante a sombra, porém, seu desenvolvimento e crescimento, só é efetivado e acelerado quando há disponibilidade de luz.

Para Carapa guianensis (Figura 3 C) a estrutura diamétrica se comportou como relatado por Schwartz et al. (2008), com um padrão decrescente e com maiores densidades nas fases de plântulas e jovens das árvores. Esta distribuição apresentada pela espécie sugere que a mesma conseguiu se estabelecer bem na floresta até o centro de classe $45 \mathrm{~cm}$, sendo que as maiores classes apresentaram densidade bastante inferior.

A distribuição diamétrica da espécie Couratari sp. seguiu a tendência da curva do tipo "J invertido", com 96,81\% das árvores presentes até o centro de classe diamétrica de $45 \mathrm{~cm}$. Esta forma de distribuição das árvores nas classes de diâmetro do gênero Couratari, também foi relatado por Procópio et al. (2010) para os municípios de Manaus e Belterra, em áreas de Floresta Ombrófila Densa. Percebe-se que Couratari sp. possui boa regeneração, mas a presença de árvores com maiores diâmetros (DAP > $50 \mathrm{~cm}$ ) é escassa.
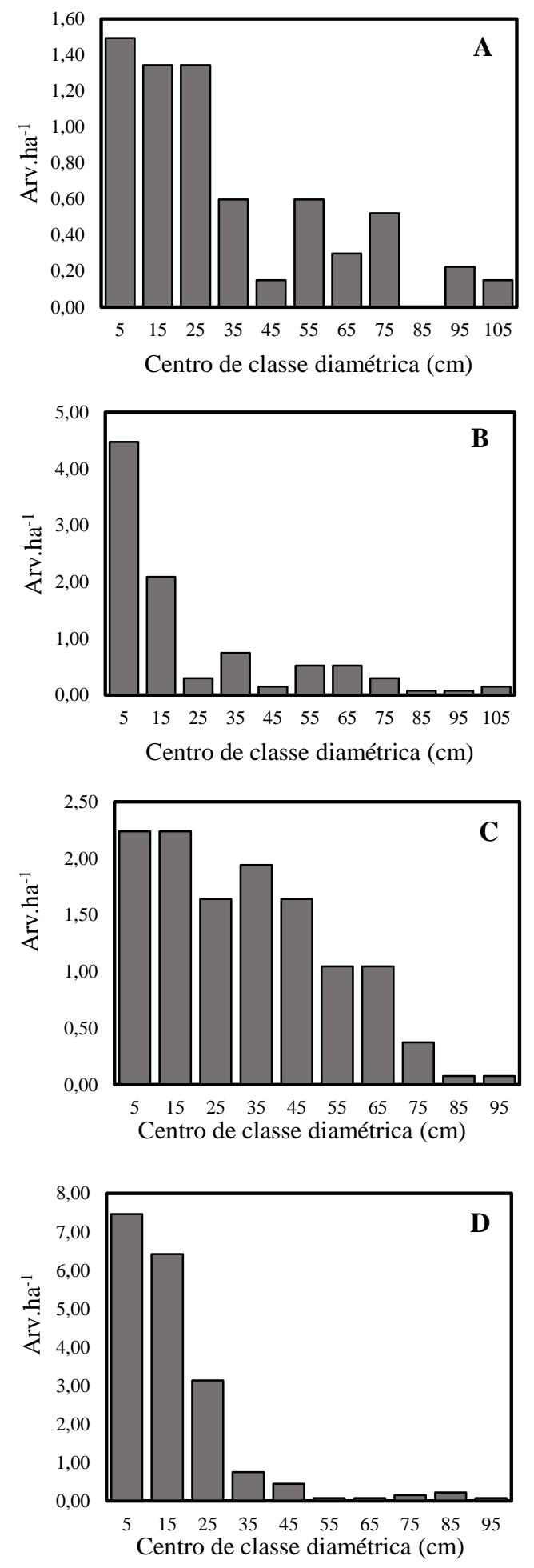

Figura 3. Distribuição diamétrica das espécies com grande relevância econômica na região, dentre as espécies de maiores valores de importância, sendo elas: (A) Lecythis lurida, (B) Manilkara elata, (C) Carapa guianensis e (D) Couratari sp. em uma área não manejada na Flona do Tapajós, PA.

\section{Distribuição volumétrica}

O volume estimado para a área de estudo foi de 233 $\mathrm{m}^{3} \cdot \mathrm{ha}^{-1}$, sendo os maiores volumes concentrados nos 
centros de classes de $15 \mathrm{~cm}$ a $75 \mathrm{~cm}$ (Figura 4). As árvores com DAP $\geq 50 \mathrm{~cm}$ apresentaram um volume de $130 \mathrm{~m}^{3} \cdot \mathrm{ha}^{-1}$. As 10 espécies com DAP $\geq 50 \mathrm{~cm}$ que apresentaram os maiores volumes foram: Hymenaea courbaril, Carapa guianensis, Manilkara elata, Bertholletia excelsa, Lecythis lurida, Virola melinonii, Tachigali chrysophylla, Tachiagli sp., Eschweilera sp., Nectandra sp. Dentre estas, as espécies Hymenaea courbaril, Carapa guianensis, Manilkara elata e Lecythis lurida, bastante almejadas no mercado florestal, somaram juntas $41,45 \mathrm{~m}^{3} \cdot \mathrm{ha}^{-1}$.

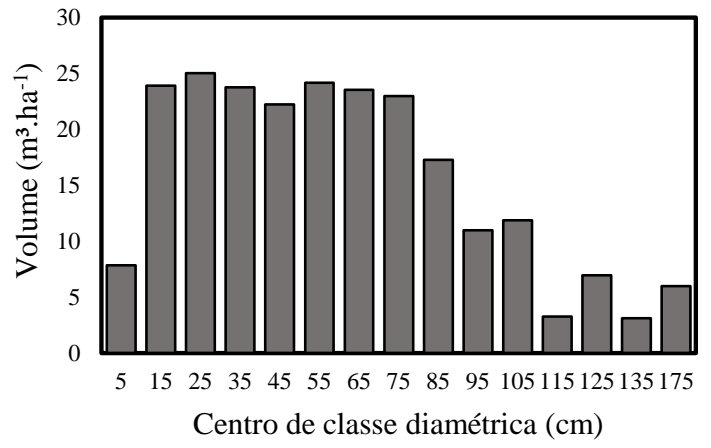

Figura 4. Distribuição volumétrica dos indivíduos em uma área não manejada na Floresta Nacional do Tapajós, PA.

O conhecimento da distribuição volumétrica de uma floresta em classes diamétricas se faz importante, principalmente para conhecer o potencial para um possível manejo florestal. No entanto, muitas vezes isto não é considerado, sendo que Putz (2000) já alertava para esta falha nos planos de manejo florestal, e ressaltava que a obtenção dos recursos das florestas com enfoque para a madeira, deveriam estar no mínimo vinculados ao conhecimento da produção da floresta em classes de diâmetro.

\section{Qualidade de fuste e presença de cipó titica (Heteropsis spp.)}

Foi observado maior ocorrência de árvores com qualidade de fuste do tipo 2, ou seja, árvores que possuem pequena tortuosidade ao longo de seu fuste (Tabela 2). Considerando somente o estoque de colheita (árvores com DAP $\geq 50 \mathrm{~cm}$ ) que corresponde a 288 árvores ou 21,50 arv.hat ${ }^{-1}$, um total de $89,58 \%$ dos indivíduos apresentaram fuste passíveis de comercialização.

Tabela 2. Número de árvores de acordo com a classificação da qualidade de fuste e seu percentual para todas as árvores e para aquelas com DAP $\geq 50 \mathrm{~cm}$, em uma área não manejada na Floresta Nacional do Tapajós, PA

\begin{tabular}{cccc}
\hline $\begin{array}{c}\text { Qualidade de fuste } \\
(\mathbf{Q F})\end{array}$ & Total de árvores & $(\boldsymbol{\%})$ & $\begin{array}{c}\text { DAP } \geq \mathbf{5 0} \mathbf{~ c m} \\
(\boldsymbol{\%})\end{array}$ \\
\hline 1 & 423 & 15,48 & 25,69 \\
2 & 1875 & 68,63 & 63,89 \\
3 & 434 & 15,89 & 10,42 \\
\hline Total Geral & 2732 & 100,00 & 100,00 \\
\hline
\end{tabular}

As QF1 e QF2 são aceitáveis no mercado local, com preferência para as árvores com QF1. Considerando os níveis de futura colheita (CT2: 10,0 $\mathrm{cm} \leq \mathrm{DAP}<50,0$ $\mathrm{cm})$ e colheita (CT3: DAP $\geq 50 \mathrm{~cm})$, tem-se um total de 269,40 arv ha $^{-1}$, podendo indicar potencial para colheita de madeira.

Foram registradas 143 árvores hospedeiras do cipó titica (Heteropsis spp.), com uma densidade de 26,42 arv.ha ${ }^{-1}$, distribuídas em 64 espécies. Foram totalizados 607 cipós maduros e 530 cipós verdes. As dez espécies com maior incidência de cipó-titica estão presentes na tabela 3 .

Tabela 3. Relação da incidência de cipó-titica nas dez espécies com maior ocorrência em cipós maduros e verdes, inventariadas em sem intervenção antrópica na Floresta Nacional do Tapajós, PA.

\begin{tabular}{|c|c|c|c|}
\hline Espécie & $\begin{array}{c}\text { Cipó } \\
\text { Maduro }\end{array}$ & Espécie & $\begin{array}{l}\text { Cipó } \\
\text { Verde }\end{array}$ \\
\hline $\begin{array}{l}\text { Lecythis lurida } \\
\text { (Miers) S.A.Mori }\end{array}$ & 45 & $\begin{array}{l}\text { Couratari oblongifolia } \\
\text { Ducke \& Kunth }\end{array}$ & 38 \\
\hline $\begin{array}{l}\text { Nectandra } \\
\text { rubra }(\mathrm{Mez}) \\
\text { C.K.Allen }\end{array}$ & 32 & Protium paniculatum Engl. & 34 \\
\hline Virola cuspidata & 31 & $\begin{array}{l}\text { Tachigali chrysophylla } \\
\text { (Poepp.) Zarucchi \& } \\
\text { Herend. }\end{array}$ & 27 \\
\hline Eschweilera odorata & 31 & $\begin{array}{l}\text { Rinorea guianensis var. guia } \\
\text { nensis (Aubl.) Sw. }\end{array}$ & 25 \\
\hline $\begin{array}{l}\text { Tachigali } \\
\text { chrysophylla (Poepp.) } \\
\text { Zarucchi \& Herend. }\end{array}$ & 30 & $\begin{array}{l}\text { Licania brittoniana var. hete } \\
\text { romorpha Fritsch }\end{array}$ & 24 \\
\hline $\begin{array}{l}\text { Protium paniculatum } \\
\text { Engl. }\end{array}$ & 26 & Ormosia paraenses Ducke & 24 \\
\hline $\begin{array}{l}\text { Couratari oblongifolia } \\
\text { Ducke \& Kunth }\end{array}$ & 21 & Virola cuspidata & 18 \\
\hline $\begin{array}{l}\text { Protium heptaphyllum } \\
\text { var. angustifolium (A } \\
\text { ubl.) Marchand }\end{array}$ & 20 & $\begin{array}{l}\text { Lecythis lurida (Miers) } \\
\text { S.A.Mori }\end{array}$ & 16 \\
\hline Virola duckei A.C.Sm & 20 & $\begin{array}{l}\text { Nectandra rubra }(\mathrm{Mez}) \\
\text { C.K.Allen }\end{array}$ & 15 \\
\hline $\begin{array}{l}\text { Heteromorpha var. het } \\
\text { eromorpha Benth. }\end{array}$ & 18 & Coussarea paniculata Standl & 15 \\
\hline Subtotal & 319 & & 281 \\
\hline Total & 607 & & 530 \\
\hline
\end{tabular}

Santos et al. (2018) também registraram como principais espécies em associação com cipó titica Tachigali chrysophylla e Protium heptaphyllum na Flona do Tapajós. Contudo, a densidade de árvores hospedeiras registradas pelos autores foi inferior a deste este estudo, sendo 2,7 arv.ha ${ }^{-1}$. Um estudo realizado por Bentes Gama et al. (2013) no município de Machadinho do Oeste, RO encontrou 268,66 arv.ha-1 que hospedam Heteropsis spp.

As árvores hospedeiras apresentaram altura comercial variando de 1 a $25 \mathrm{~m}$. Foi observado que a classe de altura de 12,5 m, concentrou maior quantidade de árvores hospedeiras (Figura 6). Com base na distribuição dos hospedeiros em classes de altura, nota-se que as árvores do estrato médio da floresta, hospedam em maior quantidade o cipó titica.

De acordo com Ribeiro et al. (2013), em áreas com destinação ao manejo é necessário a aplicação de tratamentos silviculturais, como o corte de cipós, para diminuir os riscos de acidentes, durante as operações de colheita e queda de outras árvores indesejadas. No entanto, pode ser possível aliar o manejo madeireiro com o manejo de recursos não madeireiros em áreas potenciais, como no caso desta área que possui boa ocorrência de cipó comercial, principalmente devido à importância deste cipó para comunidades da região, pois dele são confeccionados diversos produtos (Santos et al. 2018).

O mercado regional de Santarém - PA, município vizinho da Flona do Tapajós, absorve os produtos elaborados deste cipó, com alguns pontos de comercialização, principalmente em estabelecimentos de vendas de artesanatos (Santos et al. 2018). A venda dos produtos tem o período de alta temporada, com a chegada dos turistas na região e em datas comemorativas (Ferreira e Bentes-Gama 2005), o que fornece maiores lucros para esta atividade em determinadas épocas do ano. 


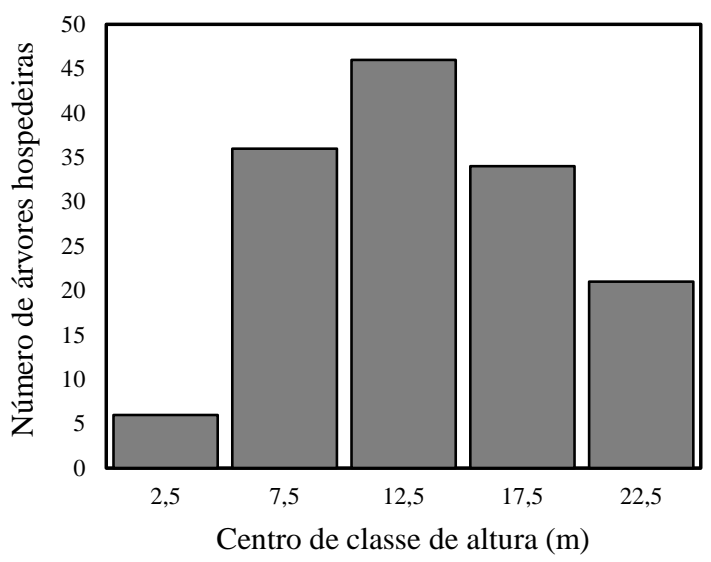

Figura 6. Distribuição das árvores hospedeiras do cipó titica em classes de altura, em uma área não manejada na Floresta Nacional do Tapajós, PA.

Nota-se então, que a floresta em estudo pode proporcionar outras opções de manejo, como a do cipó titica. Devido à densidade de árvores hospedeiras e a quantidade de cipós verdes e maduros encontrados, temse bom potencial para utilização deste produto não madeireiro como uma alternativa de renda às comunidades existentes na Flona do Tapajós e regiões vizinhas.

\section{Conclusão}

A floresta apresentou estoque de crescimento e de árvores de maior porte, destacando a ocorrência de algumas espécies com boa aceitação no mercado, dentre as quais apresentaram maiores volumes na classe de estoque comercial. A maioria das espécies que compõem estes estoques têm qualidade de fuste exigida para comercialização.

A área de estudo apresenta potencial de recursos madeireiros e não madeireiros, elucidando que o uso múltiplo de florestas deve ser considerado e estudos que mostrem esta viabilidade devem ser incentivados e replicados na Amazônia.

\section{Referências}

Alvares CA, Stape, JL, Sentelhas PC, Gonçalves JLM, Sparovek G (2013) Köppen's climate classification map for Brazil. Meteorologische Zeitschrift, 22 (6): 711-728.

Andrade DF, Gama JRV, Melo LO, Ruschel AR (2015) Inventário florestal de grandes áreas na Floresta Nacional do Tapajós, Pará, Amazônia, Brasil. Biota Amazônia, 5(1): 109-115.

Andrade RTG, Manzatto AMA (2014) Insuficiência de Politicas Públicas Nacionais Pró- Biodiversidade Amazônica. Revista Gestão e Políticas Públicas, 4(2): 219-239.

Begon M, Harper JL, Towhsend CR (2006) Fundamentos em Ecologia. Porto Alegre: Artmed, 592p.

Bentes-Gama MM, Scolforo JRS, Gama, JRV (2002) Potencial produtivo de madeira e palmito de uma floresta secundária de várzea baixa no estuário amazônico. Revista Árvore, 26(3): 311-319.

Bentes-Gama, M. M, Vieira, AH, Rocha, RB. (2013) Ecological features of titica vine (Heteropsis flexuosa (Kunth) GS Bunting) in Rondônia State, Northwest
Brazilian Amazon. Anais da Academia Brasileira de Ciências, 85(3): 203-211.

Brower JE, Zar J.H (1984) Field \& laboratory methods for general ecology. W.C. Brown Publishers, Boston.

Brower SE, Zarr JH, Ende CN (1997) Field and laboratory methods for general ecology. 4th edition. McGraw Hill, Boston.

Calegario N et al. (1993) Parâmetros florísticos e fitossociologicos da regeneração natural de espécies arbóreas nativas no sub-bosque de povoamentos de Eucalyptus. Revista Árvore, 17(1): 16-29.

Callegaro RM et al. (2012) Estrutura do componente arbóreo de uma floresta estacional decidual ripária em Jaguari, RS. Ciência Rural, 42(2): 306-311.

Camarero, JJ, Gutiérrez E, Fortin M (2000) Spatial pattern of subalpine Forestalpine grassland ecotones in the Spanish Central Pyrenees. Forest Ecology and Management, 134: 1-16

Capretz RL (2004) Análise dos padrões espaciais de árvores em quatro formações florestais do Estado de São Paulo, através de análises de segunda ordem, como a função de K de Ripley. Dissertação, Escola Superior de Agricultura Luiz de Queiroz/ESALQ, Piracicaba, 79 p.

Costa DHM. Carvalho JOP, Berg EVD (2007) Crescimento diamétrico de maçaranduba (Manilkara Huberi Chevalier) após a colheita de madeira. Amazônia: Ciência \& Desenvolvimento, 3(5): 65-76.

Espírito-Santo FDB, Shimabukuro YE, Aragão LEOC, Machado ELM (2005) Análise da composição florística e fitossociológica da floresta nacional do Tapajós com o apoio geográfico de imagens de satélites. Acta Amazonica, 35(2):155- 173.

Ferreira MGR, Bentes-Gama MM (2005) Ecologia e formas de aproveitamento econômico do cipó-titica (Heteropsis flexuosa (H. B. K.) G. S. Bunting). Porto Velho, EMBRAPA Rondônia. (Boletim Técnico, 95), $22 \mathrm{p}$.

Flora do Brasil 2020 em construção (2018) Jardim Botânico do Rio de Janeiro. Disponível em: < http://floradobrasil.jbrj.gov.br/ >. Acesso em: 06 Out 2018.

Fontana C, Sevegnani L (2012) Quais são as espécies arbóreas comuns da floresta estacional decidual em Santa Catarina? Revista de Estudos Ambientais, 14: 74-88

Gama JRV, Bentes-Gama MM, Scolforo JRS (2005) Manejo sustentado para floresta de várzea na Amazônia oriental. Revista Árvore, 29(5): 719-729.

Gonçalves FG, Santos JR (2008) Composição florística e estrutura de uma unidade de manejo florestal sustentável na Floresta Nacional do Tapajós, Pará. Acta Amazonica, 38(2): $229-244$.

Heinsdijk D, Bastos AM (1963) Inventários florestais na Amazônia. Boletim do Setor de Inventário Florestal, 6: 110.

Instituto Brasileiro de Geografia e Estatística - IBGE (2012) Manual técnico da vegetação brasileira. Rio de Janeiro: $275 \mathrm{p}$. 
Lehn CR, Resende UM (2007) Estrutura populacional e padrão de distribuição espacial de Cyathea delgadii Sternb. (Cyatheaceae) em uma Floresta Estacional Semidecidual no Brasil Central. Biociências, 13(4): 188195.

Lima-Filho DA, Revilla J, Coelho LS, Ramos JF, Santos JL, Oliveira JG (2002) Regeneração natural de três hectares de floresta ombrófila densa de terra firme na região do rio Urucú, Amazonas, Brasil. Acta Amazônica. 32(4): 555-570.

Lima RBA, Silva JAA, Marangon LC, Ferreira RLC., Silva RKS (2012) Fitossociologia de um trecho de floresta ombrófila densa na Reserva de Desenvolvimento Sustentável Uacari, Carauari, Amazonas. Scientia plena, 8(1): $1-12$.

Mello MAR (2002) Distribuição espacial de plantas em florestas Neotropicais. Monografia, Universidade Estadual de Campinas, $15 \mathrm{p}$

Mendonça AP, Ferraz IDK (2007) Óleo de andiroba: processo tradicional da extração, uso e aspectos sociais no estado do Amazonas, Brasil. Acta Amazonica, 37: 353-364.

Oliveira NA, Amaral IL (2004) Florística e fitossociologia de uma floresta de Vertente na Amazônia Central, Amazonas, Brasil. Acta Amazônica, 34(1): 2134.

Procópio LC, Gayot M, Sist P, Ferraz IDK (2010) As espécies de tauari (Lecythidaceae) em fl orestas de terra firme da Amazônia: padrões de distribuição geográfica, abundâncias e implicações para a conservação. Acta botânica brasilica, 24(4):883-897.

Putz FE, Dykstra DP, Heinrich R (2000) Why poor logging practices persist in the tropics. Conservation Biology, 14(4): 951-956.

Reinhardt G, Rettenmaier N, Gãrtner S, Pastowski A (2007) Rain forest for biodiesel?: ecological effects of using palm oil as a sourse of energy. Frankfurt: WWF Germany.

Ribeiro RBS, Gama JRV, Martins SV, Moraes A, Santos CAA, Carvalho AN (2013) Estrutura florestal em projeto de assentamento, comunidade São Mateus, município de Placas, Pará, Brasil. Ceres, 60(5): 610-620.

Salomão RP, Santana AC, Brienza Júnior S, Gomes VHF (2012) Análise fitossociológica de floresta ombrófila densa e determinação de espécies-chave para recuperação de área degradada através da adequação do índice de valor de importância. Boletim do Museu Paraense Emílio Goeldi. Ciências Naturais, Belém, 7(1): 57-102.

Santos LE, Gama JRV, Ribeiro RBS, Silva AA, Cruz GS, Rodrigues BL (2018) Inventário de cipó titica na Floresta Nacional do Tapajós e comercialização em Santarém, Pará. Advances in Forestry Science, 5(2): 309-314.

SEMAS, Secretaria de Estado de Meio Ambiente e Sustentabilidade (2015) Instrução Normativa $N^{o} 5$.

Shanley P (2005) Andiroba (Carapa guianensis, Aublet.). In: SHANLEY, P.; MEDINA, G. Frutíferas e plantas úteis na vida amazônica. Belém: Cifor. p. 41-50.

Schwartz G, Nascimento NA, Menezes AJEA (2008) Estrutura populacional de espécies de interesse florestal não-madeireiro no sudeste do Pará, Brasil. Amazônia: Ciência e Desenvolvimento, 4(7): 117-130.

Silva-Junior MC (2005) Fitossociologia e estrutura diamétrica na mata de galeria do Pitoco, na Reserva Ecológica do BGE, DF. Cerne. 11(2): 147-158.

Silva VSM (2006) Manejo de florestas nativas: planejamento, implantação e monitoramento. Universidade Federal do Mato Grosso. Cuiabá, 114p.

Soares CPB, Rodello CM, Souza AL, Leite HG, Soares VP, Silva GF (2009) Comparação entre procedimentos de amostragem para espécies florestais raras e padrão de distribuição espacial agregado. Revista Árvore, 33(3): 545-553.

Souza DR, Souza AL, Leite HG, Yared JAG (2006) Análise estrutural em Floresta Ombrófila Densa de Terra Firme não explorada, Amazônia Oriental. Árvore, 30(1): 75-87.

Souza AL, Soares CPB (2013) Florestas nativas: estrutura, dinâmica e manejo. Ed. UFV.

Thompson SK, Seber GAF (1996) Adaptive sampling. New York: John Wiley \& Sons, 265p.

Vibrans AC, Sevegnani L, Lingner DV, Gasper AL, Sabbagh S (2010) Inventário florístico florestal de Santa Catarina (IFFSC): aspectos metodológicos e operacionais. Pesquisa Florestal Brasileira, 30: 291-302.

Vieira DS, Gama JRV, Andrade DFC (2014) Estrutura populacional e padrão de distribuição espacial de Pouteria cladantha Sandwith em uma floresta sob regime de manejo sustentável, Pará. Biota Amazonia, 4(3): 4247. 